\title{
Salmonella Infection after Surgery of Small Intestine Foreign Bodies
}

\author{
Jiun-Nong Lin ${ }^{a}$ b Chung-Hsu Lai ${ }^{a}$ Chun-Kai Huang ${ }^{a}$ Hsing-Chun Chung ${ }^{a}$ \\ Shiou-Haur Liang ${ }^{a}$ Hsi-Hsun Lin ${ }^{a}$ \\ aDivision of Infectious Diseases, Department of Internal Medicine, E-Da Hospital/I-Shou University, \\ Yan-Chau Shiang, and ${ }^{b}$ Graduate Institute of Medicine, Kaohsiung Medical University, Kaohsiung, Taiwan
}

\section{Key Words}

Salmonella $\cdot$ Salmonella choleraesuis $\cdot$ Salmonella enterica

serotype choleraesuis $\cdot$ Small intestine

\begin{abstract}
Objective: To report the first case of Salmonella enterica serotype choleraesuis infection after surgery for small intestine foreign bodies. Clinical Presentation and Intervention: A 52-year-old woman presented to our hospital with the chief complaint of left abdominal pain for 1 day. The plain kidney-ureter-bladder film and abdominal computed tomography scan showed foreign bodies in the peritoneum. Metallic foreign bodies in the jejunum were found during surgery. Following surgery, the patient developed fever, and blood culture yielded Salmonella enterica serotype choleraesuis. The patient recovered smoothly after antibiotic therapy. Conclusion: Salmonella enterica serotype choleraesuis is a highly invasive serotype of nontyphodial Salmonella. In addition to gastroenteritis, bacteremia, or extraintestinal localized infections, physicians should know that it could complicate intestinal surgery.

Copyright $\odot 2009$ S. Karger AG, Basel
\end{abstract}

\section{Introduction}

Nontyphoidal Salmonella species are major causes of foodborne infections, and can lead to self-limited acute gastroenteritis, bacteremia, or extraintestinally localized infections involving many organs. Young children and patients with certain underlying conditions, including acquired immunodeficiency syndrome, malignancies, hemolytic anemia, and inflammatory bowel disease, or those receiving immunosuppressive therapy, are at increased risk of Salmonella infections [1-3]. Among more than 2,000 nontyphoidal serotypes, Salmonella enterica serotype choleraesuis shows a much higher predilection for causing bacteremia and invasive extraintestinal infections in humans [1-3]. Postoperative infection with Salmonella has been reported in rare cases of orthopedic prostheses replacement surgery and intracranial surgery [4-6]. However, such an infection has never been reported resulting from intestinal surgery. Here, we report a patient who received surgery for foreign bodies in the small intestine that was subsequently complicated with an infection by $S$. enterica serotype choleraesuis. The purpose of this case report is to alert physicians to the fact that invasive salmonellosis could complicate surgery of the small intestine.

\section{KARGER \\ Fax +4161306 1234 \\ E-Mail karger@karger.ch}

www.karger.com
(C) 2009 S. Karger AG, Basel

1011-7571/09/0183-0242\$26.00/0

Accessible online at:

www.karger.com/mpp
Hsi-Hsun Lin

Division of Infectious Diseases, Department of Internal Medicine

E-Da Hospital/I-Shou University, 1 E-Da Road

Jiau-Shu Tsuen, Yan-Chau Shiang, Kaohsiung County 824 (Taiwan)

Tel. +886 7615 0011, ext. 91469, Fax +886 7615 0928, E-Mail erlongtw@yahoo.com.tw 
1

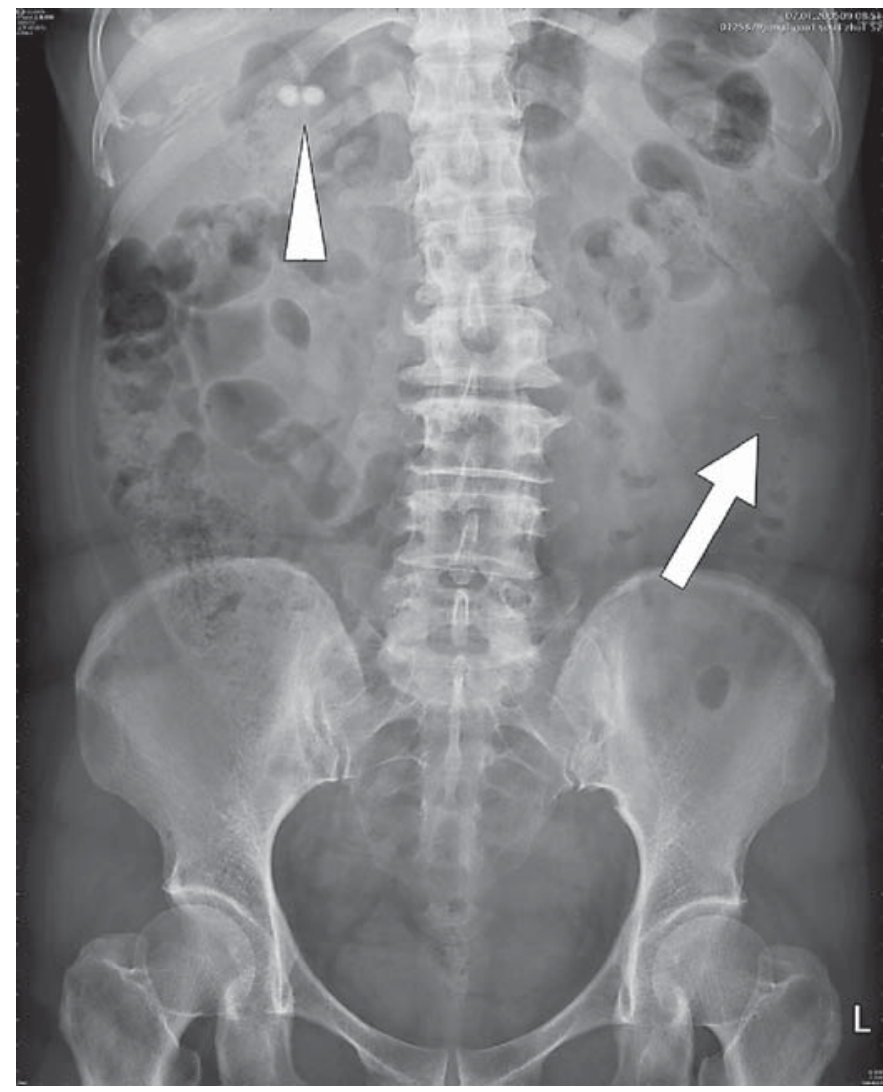

$\mathrm{L}$

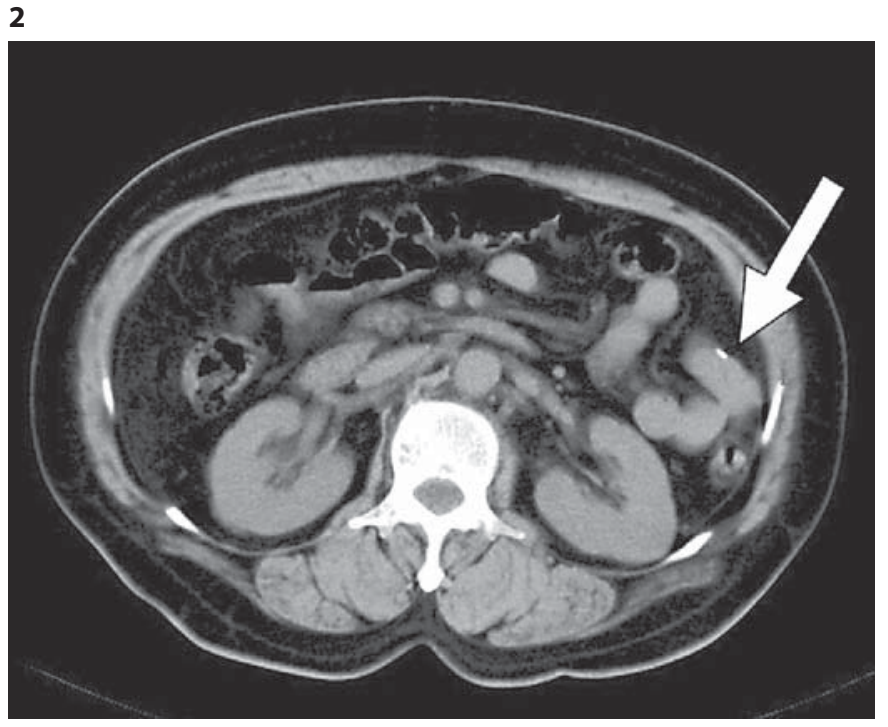

Fig. 1. Plain kidney-ureter-bladder film showed 2 radiopaque materials over the left abdomen (arrow) and 2 calcified stones over the right upper quadrant (arrowhead).

Fig. 2. Abdominal CT scan showed metallic material at the small intestine (arrow).

\section{Case Report}

A 52-year-old woman presented to the Emergency Department, E-Da Hospital, a university-affiliated hospital in southern Taiwan, with the chief complaint consisting of abdominal pain on the left side for 1 day. She had no known systemic diseases and had been healthy until the day prior to admission. On arrival, she was clear and her vital signs were blood pressure: 146/70 mm Hg; pulse rate: 78 beats/min; respiratory rate: 20 breaths/min; and body temperature: $36.8^{\circ} \mathrm{C}$. Physical examination revealed tenderness over the left paraumbilical region of the abdomen. Peritoneal signs with muscle guarding and rebounding pain were both disclosed when pressure was applied to the tender point of the abdomen. Her bowel sound decreased on auscultation. Laboratory findings revealed a red blood cell count of $4.52 \times 10^{6} / \mathrm{mm}^{3}$, hemoglobin: $13.2 \mathrm{~g} / \mathrm{dl}$, hematocrit: $40.2 \%$; white blood cell count: $8,970 / \mathrm{mm}^{3}$; platelets: $213,000 / \mathrm{mm}^{3}$; aspartate aminotransferase: $21 \mathrm{IU} / \mathrm{l}$; creatinine: $0.9 \mathrm{mg} / \mathrm{dl}$; sodium: $142 \mathrm{mmol} / \mathrm{l}$; potassium: 3.8 $\mathrm{mmol} / \mathrm{l}$; blood sugar: $117 \mathrm{mg} / \mathrm{dl}$; amylase: $34 \mathrm{U} / \mathrm{l}$ (normal range: 0-220 U/1); and lipase: $100 \mathrm{U} / 1$ (normal range: 0-190 U/1). A plain radiograph of abdomen showed 2 radiopaque materials over the left abdomen (fig. 1). The lateral view confirmed these 2 radiopaque materials were within the abdomen. An abdominal computed tomography (CT) scan was performed and revealed 2 linear metallic foreign bodies at the jejunum (fig. 2), but no free air in the peritoneum. Along with the foreign bodies, gallstones and bilateral ovarian cystic masses were also discovered in the CT scan. The patient did not recall ingesting any metallic materials. Under the impression that this was an instance of jejunal foreign bodies with peritonitis, a laparotomy was performed and revealed 2 metallic foreign bodies which had penetrated the jejunum and been encased on the serosa of jejunum $100 \mathrm{~cm}$ from Treitz's ligament. The foreign bodies were removed, the serosa and jejunum debrided, and a primary suture of the jejunum performed. A large left ovarian hemorrhagic simple cyst with torsion was also found, and a left oophorectomy executed. A Jackson-Pratt drain tube was left in the cul-de-sac after the surgery.

The patient experienced a high fever, of up to $40^{\circ} \mathrm{C}, 4$ days after surgery. Cefepime (Maxipime; Bristol-Myers Squibb) was given ( 2 g every $12 \mathrm{~h}$ intravenously) for the possible nosocomial infection after specimens for blood cultures were collected. Physical examination revealed discharge from the surgical wound and fascia deadhesion after removal of the sutures. A laparotomy was performed again to check for anastomotic leakage or wound infection. On direct inspection, there were no dirty ascites, pus, or anastomotic leakage, but there was a small amount of debris coating the serosa of the small intestine $390 \mathrm{~cm}$ from Treitz's ligament. Debridement of the debris and intraperitoneal irrigation with normal saline were performed. While culture of the debris was negative, the blood culture yielded $S$. enterica serotype choleraesuis. The stool culture was negative for Salmonella. The organism was susceptible to ampicillin, ceftriaxone, and trimethoprim/sulfamethoxazole, but resistant to chloramphenicol and ciprofloxacin when screened by disk diffusion. Intravenous ceftriaxone 
(Rocephin; Roche) was prescribed (1 g every $12 \mathrm{~h}$ ) and the fever subsided progressively with this treatment. The patient was discharged from the hospital 12 days after the second operation, and was in stable condition with negative follow-up blood cultures taken by the Outpatient Department.

\section{Discussion}

This is the first case report of S. enterica serotype choleraesuis infection after surgery for foreign bodies in the small intestine. S. enterica serotype choleraesuis is a hostadapted serotype that causes swine paratyphoid, and is also highly pathogenic to humans with septicemia and invasive extraintestinal manifestations $[3,7]$. However, it is typically limited to swine (which serve as a carrier and reservoir), as well as facilities that have been contaminated [3].

Although this highly invasive serotype is infrequently isolated from human sources in the USA, Canada, and the UK, it is of particular concern in Taiwan [3, 8-10]. In the reports by Chiu et al. [3] and Chen et al. [11], serotype choleraesuis ranked the second most frequent isolate of Salmonella in Taiwan. A retrospective study conducted by Chen et al. [11] found that most adult patients with serotype choleraesuis bacteremia had obvious risk factors, including malignancy, liver cirrhosis, systemic lupus erythematosus, or previous use of corticosteroids [11]. Subsequent local infections with septic arthritis, pneumonia, peritonitis, and cutaneous abscess were noticed in $21 \%$ of the bacteremic patients.

Postoperative infection with Salmonella is rarely encountered, and typically only reported in prosthetic replacement surgery and intracranial surgery [4-6]. Salmonellosis complicating a surgery for the removal of foreign bodies is very unusual.

Our patient, who came from southern Taiwan, an area endemic with $S$. enterica serotype choleraesuis, had been previously healthy with no symptoms or signs of Salmonella infection before this incident. Because the stool cultures were negative for Salmonella, there was no evidence showing our patient was a reservoir or carrier of $S$. enterica serotype choleraesuis. Although we cannot ascertain whether the source of Salmonella bacteremia was from the patient or the hospital, the source of the infecting pathogen was believed to be the result of the surgery, presumably from contaminated facilities or environment.

\section{Conclusion}

S. enterica serotype choleraesuis can cause bacteremia and various types of severe and potentially life-threatening focal infections. This infection could be complicated surgery, especially in some countries. Surgeons should beware of the possibility of $S$. enterica serotype choleraesuis bacteremia following intestinal surgery.

\section{References}

1 Huang CT, Lo CB: Human infections with Salmonella choleraesuis in Hong Kong. J Hyg 1967;65:149-163.

-2 Allison MJ, Dalton HP, Escobar MR, Martin CJ: Salmonella choleraesuis infections in man: a report of 19 cases and a critical literature review. South Med J 1969;62:593-596.

3 Chiu CH, Su LH, Chu C: Salmonella enterica serotype choleraesuis: epidemiology, pathogenesis, clinical disease, and treatment. Clin Microbiol Rev 2004;17:311-322.
4 Schröder J, Palkovic S, Kipp F, Wassmann H: Salmonella enteritidis causing brain abscess and coxitis following intracranial surgery. Acta Neurochir (Wien) 2003;145:919-921.

5 Madan S, Abbas D, Jowett RL, Mounce K: Salmonella enteritidis infection in total knee replacement. Rheumatology (Oxford) 2001; 40:112-113.

6 Chong PY, Sporer SM: Case report: Salmonella infection following total hip arthroplasty. Iowa Orthop J 2005;25:42-43.

7 Field HI: Salmonellosis in animals. Vet Rec 1958;70:1050-1052.

-8 Olsen SJ, MacKinnon LC, Goulding JS, Bean $\mathrm{NH}$, Slutsker L: Surveillance for foodbornedisease outbreaks - United States, 19931997. MMWR CDC Surveill Summ 2000;49: $1-62$.
-9 Tauxe RV: Emerging foodborne diseases: an evolving public health challenge. Emerg Infect Dis 1997;3:425-434.

10 Barrel RA: Isolations of salmonellas from humans and foods in the Manchester area: 1981-1985. Epidemiol Infect 1987;3:277284.

-11 Chen YH, Peng CF, Tsai JJ, Hwang KP, Lu PL, Cheng HH, Peng CF: Epidemiological study of human salmonellosis during 1991-1996 in southern Taiwan. Kaohsiung J Med Sci 1999; 15:127-136. 